\title{
Sur l'emploi d'échelles verticales et horizontales différentes dans les études sur modèles réduits en hydraulique
}

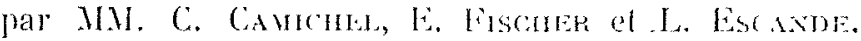

Dans l'élude sur modèles réduits des phénomènes d'hydraulique fluviale ou maritime, il est d'une pratique courante d'adopter des échelles horizontales el verticales très différentes; tela résulte de l'obligation où l'on se trouve d'employer une thelle horizontale assez faible pour limiter l'encombrement du modèle, tout en maintenant dans celur-ci un tirant d'eau suffisant; en effet, si la profondeur devenait trop petite, la viscosité interviendrait pour perturber l'écoulement et détruire la similitude hydraulique, et il pourrait mène arriver qu'au mouvement turbulent existant dans la nature, corresponde dans le modèle un mouvement laminaire sans aucun rapport avec celui-ci.

Avec ces échelles verticales ef horizontales différentes, il n'y a plus de similitude géonétrique entre l'ouvrage et son modèle; celui-ci devient une représentation conventionnelle de l'ouvrage à étudier et il n'existe aucune raison théorique qui permetle d'affirmer à priori, que les nouvements hydrauliques existant dans le modèle, seront la représentation, sur les mèmes bases conventionnelles, des phénomènes susceptibles de se produire dans l'ouvrage.

Plusieurs essais, effectués suivant cette méthode, en parliculier les études de l'estuaire de la Mersey failes par Osborne REY voLDs, ont indiqué certaines concordances entre les phénomènes constatés dans la nalure et les résultats des expériences de laboratoire.

Toulefois, il n'a jamais été-fait d'expérience précise permettant de se rendre comple de la validité de ce mode opératoire, ce qui est d'autant plus étonnant que celui-ci est depuis longtemps d'usage courant dans tous les laboratoires. C'est pourquoi nous avons entrepris des expériences dans ce but; nous allons indiquer les premiers résultats que nous avons obtenus.
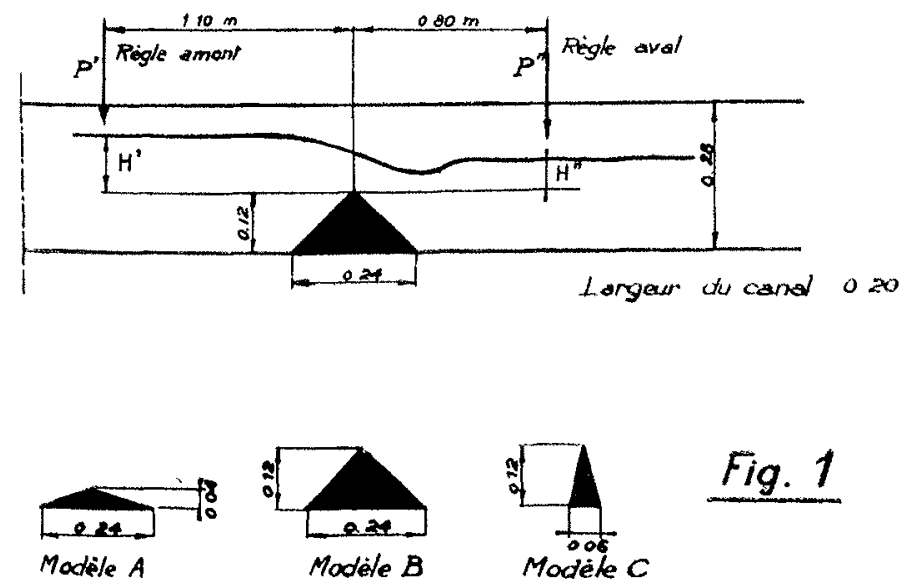

Nous avons abordé le cas particulièrement simple d'un mouvement à deux dimensions : l'écoulement plan correspondant au déversement sans contractions latérales au-dessus d'un seuil à section droite triangulaire placé en travers d'un canal d'expérience de $20 \mathrm{~cm}$. de largeur, comme l'indique la fig. 1.

Le modèle de base étant $A$, déversoir de $24 \mathrm{~cm}$. de base sur 4 de hauteur, un second modèle $B$ de 24 de base sur 12 de hauteur correspond à une même échelle horizontale et à une échelle verticale trois lois pius grande; un dernier modèle C de 6 de hase sur 12 de hauteur peut être considéré par rapport à $\mathrm{A}$, comme un nodelc ayant une échelle horzontale 4 fors moindre at une echeile vertucale truple; ce modèle peut encore être considéré comn.e le nodèle réduil à l'échelle unique $1 / 4$ d'un modèle qui, par rapport à $A$, correspondrail à une mème échelle horizontale, a à une échelle verticale 12 fois plus grande.

Les lois sem-empriques qui commandent les essais à échelles clifférentes, verlicales el horizontales, conduisent aux relations suivantes entre les quantités homologues correspondant à à ces divers modèies :

$$
\begin{aligned}
3 V \overline{3} Q_{\mathrm{A}} & =Q_{\mathrm{B}}=Q_{\mathrm{C}} \\
\mathrm{L}_{\mathrm{A}} & =\mathrm{L}_{\mathrm{B}}=\mathrm{L}_{\mathrm{C}} \\
3 \mathrm{H}_{\mathrm{A}} & =\mathrm{H}_{\mathrm{B}}=\mathrm{H}_{\mathrm{C}}
\end{aligned}
$$

$Q_{A}, Q_{B}, Q_{C}$, désignani les débits, $L_{A}, L_{B}, L_{C}$, des distances horizontales homologues, $\mathrm{H}_{\mathrm{A}}, \mathrm{H}_{\mathrm{B}}, \mathrm{H}_{\mathrm{C}}$, des distances rerticales homologues, correspondant, respectivement, aux modèles $A, B, C$.

Nous avons comparé les résullats fournis par des divers modèies dans trois séries d'expériences différentes (voir fig. 2), que notis alions caractériser par les quantités relatives au modèie B, ies grandeurs correspondant aux autres modèles se déduisant de celles-là au moyen des relations ci-dessus :

$1^{\circ}$ La charge amont au-dessus du seuil, mesurée avec la pointe 1', (fig. 1), a la valeur $\mathrm{H}_{\mathrm{B}}^{\prime}=12 \mathrm{~cm}$. la retenue aval ćlant caracténsée par la hauleur $\mathrm{H}_{\mathrm{B}}^{\prime \prime}=6 \mathrm{~cm}$. au-dessus du

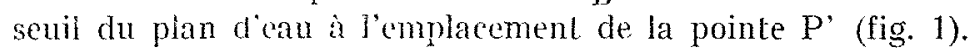

Le débit correspondant élail alors $\mathrm{Q}_{\mathrm{B}}=16,8 \overline{\mathrm{j}} \mathrm{\textrm {il }} / \mathrm{sec}$.

En prenant des charges honologues pour les modèles A el C. nous avons trouvé pour les débits correspondants, des valeurs iclles que $Q_{\mathrm{C}}=16,4 \overline{\mathrm{j}} \mathrm{hil} / \mathrm{sec}$. el $3 \times V, \overline{3} \times \mathrm{Q}_{\mathrm{A}}=17,4$ lit $/ \mathrm{sec}$. ()n voit que ha ioi des débils est approximativement vérifiée.

Par contre, en comparant le profil de la surface libre d'une part, et de ì surface de discontinuité limitant le dessous de la lame dérersante, d'autre part, dans les divers modèles, compte tenu des différentes échelles, on troure des courbes très nellemenl distinctes les unes des autres ; les trajecloires n'obéissent donc pas à ia convention géométrique imposée au modèle.

20 Lne seconde série d'expériences était caractérnsée, dans le cas du modile $\mathrm{B}$; par l'écoulement d’un débit $\mathrm{Q}_{\mathrm{B}}=12$ litsec. la relomte aval étant leile que la charge mesurée en P' soil $\mathrm{H}_{\mathrm{B}}=12 \mathrm{~cm}$. l'écoulement se faisant en nappe ondulée aves une déniveliation insignifiante entre l'amont et l'aval du modèle. lin opérant lans des conditions homologues avec les modeles A et $C . .$, nous avons trouvé que les surfaces de discontinulé inféritures, conncidarent sensiblement dans les trois modèles ; en ce qui concerne les surfaces libres, il y avait coincidence approxmative entre les modeles $A$ et $C$, le modele $B$ donnant une surface nettement inférieure.

$3^{\circ}$ En dénoyant complètement l'aval du nodèle, el réglant la charge amont à la valeur $\mathrm{H}_{B}^{\prime}=12 \mathrm{~cm}$. nous avons trouvé comme valeur du débit $\mathrm{Q}_{\mathrm{B}}=18,1 \mathrm{int} / \mathrm{sec}$. En opérant dans 
des conditions homologues avec les modèles $\mathrm{A}$ et $\mathrm{C}$, nous avons trouvé, pour les débits qui s'écoulaient, des valeurs $Q_{\mathrm{C}}=18,35$ lit/sec. et $Q_{\mathrm{A}}$, telle que $3 \times V / \overline{3} \times Q_{\mathrm{A}}=18,8 \mathrm{lil} / \mathrm{sec}$. Ies débits, tout en étant du mème ordre, sont toutefois relativement plus forts pour le modèle A.

En ce qui concerne les surfaces libres et les surfaces de dis- éléments, comne les débits, obéissent assez bien à la loi de réduction correspondant à l'emploi d'échelles verticales et horizontales différentes, par contre, pour d'autres points, comme les surfaces examinées dans l'étude actuelle, sl n'existe guère de concordance entre les divers modèles.

Ces résultats montrent toule la complexité de celte question,
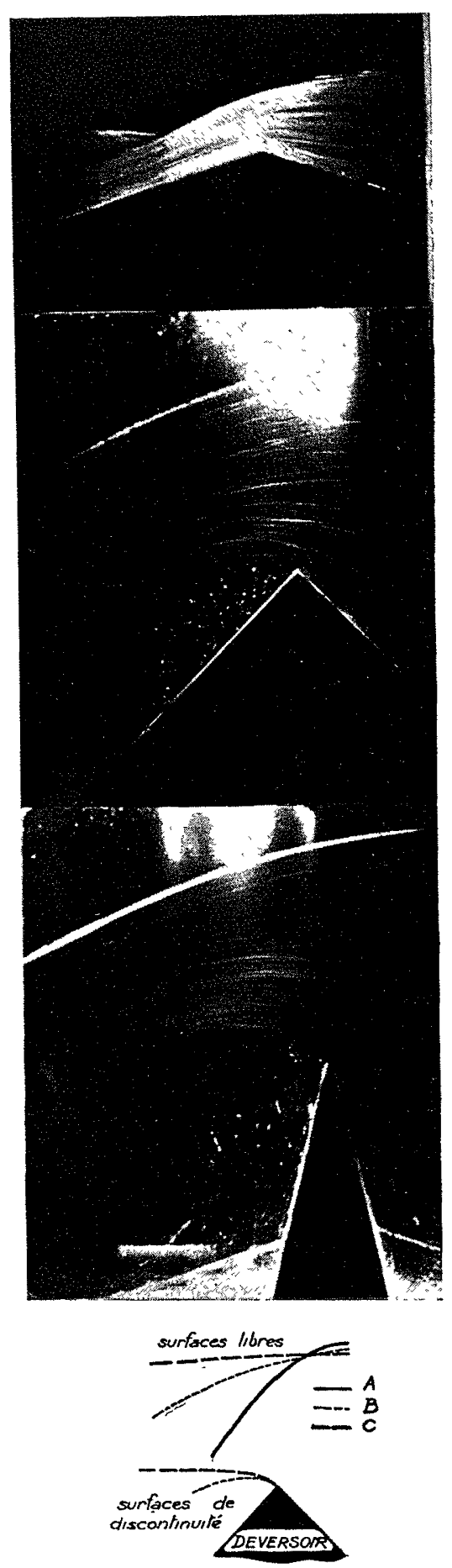

IE Expérience
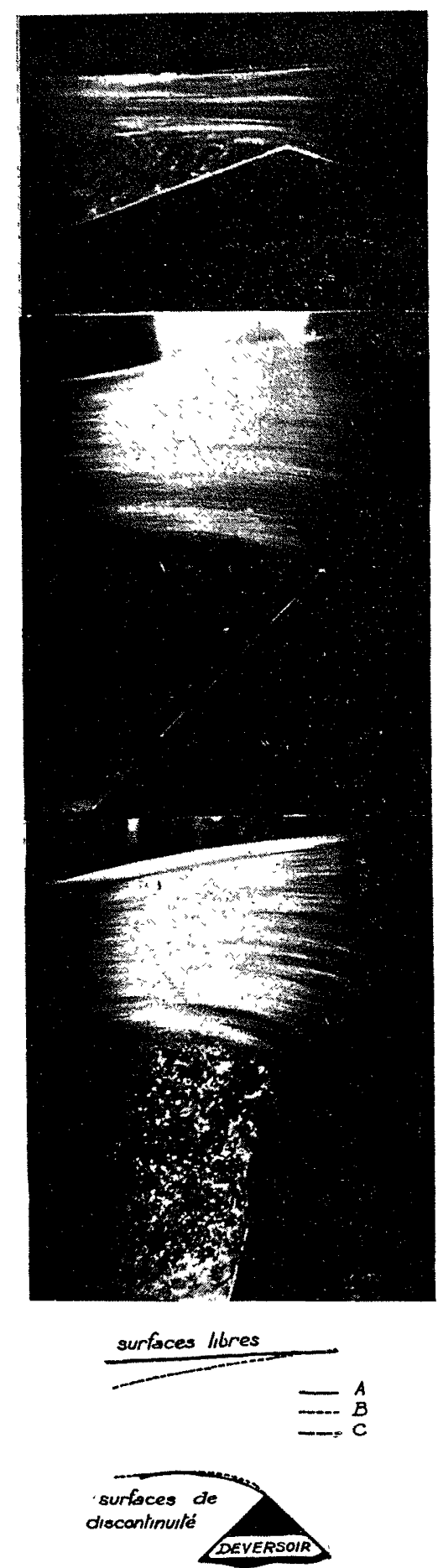

2 eme Expérience

Fig. 2
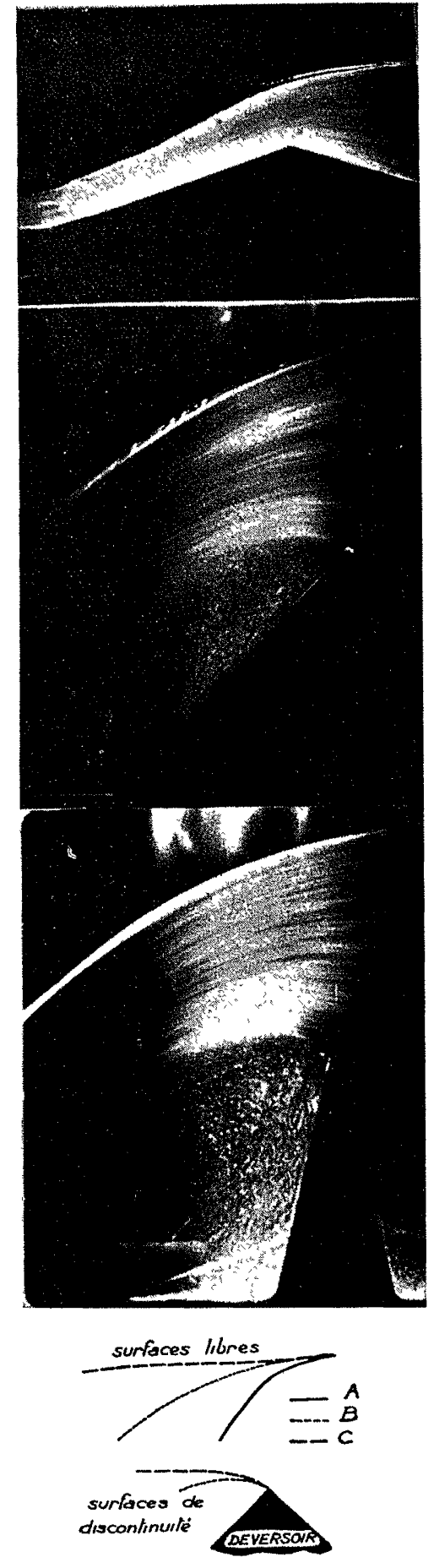

zime Expérience continuité relalives aux divers modèles, leur comparaison, à partir des conventions géométriques adoplées, montre qu'll n'existe aucune coincidence entre elles.

\section{Conclusion}

Les expériences précédentes montrent donc que, si certains dont l'intérêt est très grand en raison de son application cons tante aux études d'hydraulique fluviale el maritime, effectuée par les Laboratoires; il imporle donc de poursuivre des rechet ches systématiques dans ce domaine.

Dans une prochaine note, nous envisagerons certains point concernant les affouillements. 